\title{
A retrospective review of influences on clinicians to order whole body CT scans in trauma and its effectiveness in this regard
}

R Sloan

From London Trauma Conference 2012

London, UK. 4-7 December 2012

\section{Background}

Clinical examination alone in trauma has been shown to be ineffective, with equivocal or misleading findings in $20-50 \%$ of patients suffering from blunt polytrauma [1].

As a result, the use of whole-body CT (WBCT) in trauma assessment of patients is rapidly increasing. However, WBCT exposes patients to large amounts of radiation and so clinicians around the UK are under pressure to have strong justification for the use of WBCT. In Leeds General Infirmary (LGI) there is no specific protocol for ordering a WBCT currently in place. We aimed to assess which of the triage criteria influence the staff the most when ordering a CT - specifically: mechanism of injury (MOI) or injury severity based on clinical assessment and physiology profile. We aimed to evaluate which, if any of these factors where associated with clinically occult injury (COI) being diagnosed following WBCT.

\section{Method}

In a retrospective study, data from the trauma audit and research network and LGI's own e-records were used to assess severity of MOI (using Lerner et. al[2] to create a scale), clinical assessment severity was decided using the revised trauma score and probability of survival predicted based on this score [3]

Which of the triage criteria influenced the clinician's decision and if a clinically occult injury (COI) was found on CT were assessed and a predictive model was then used to decide which of the triage criteria had the strongest influence in diagnosing a COI.

\section{Results}

33 patients who underwent WBCT were included in the study. Mean age: 35, 31 (94\%) were men and mean injury

Royal Oldham Hospital, Oldham, UK

○ 2013 Sloan; licensee BioMed Central Ltd. This is an Open Access article distributed under the terms of the Creative Commons Attribution License (http://creativecommons.org/licenses/by/2.0), which permits unrestricted use, distribution, and reproduction in any medium, provided the original work is properly cited. severity score was $27.75 \%$ had a severe MOI, $52 \%$ had normal physiology and 55\% had severe clinical assessment. $55 \%$ had a COI found. No statistically significant relationship was found between these variables and the diagnosis of COI following WBCT. Moderate or severe MOI increased probability of COI being diagnosed by 1.368 and 4.965 respectively. Moderate and severe physiology increased the probability of diagnosing a COI by 1.368 and 8.682 respectively. Moderate clinical assessment increased the probability of diagnosing a COI by 3.526 while severe clinical assessment decreased it by $69 \%$, although results were not statistically significant.

\section{Conclusions}

Clinical assessment is primarily used by clinicians in LGI with a heavy influence of MOI. The current system is relatively accurate but further work should be done to ensure the triage system is accurate. Clinical assessment appears to be the most powerful tool even compared to imaging, however a larger study is needed to achieve statistical significance.

Published: 28 May 2013

\section{References}

1. Poletti PA, Wintermanrk M, Schynyder P, Backer CD: Traumatic injuries: the role of imaging in the management of polytrauma victim (conservative expectation). Eur Radiology 2002, 12:969-78.

2. Lerner $E B$, et al: Does mechanism of injury predict trauma center need? Prehosp Emerg Care 2011, 15:518-25.

3. Revised Trauma Score., [Online] [Cited: May 04, 2012.] http://www.trauma. org/archive/scores/rts.html.

\section{doi:10.1186/1757-7241-21-S1-S16}

Cite this article as: Sloan: A retrospective review of influences on clinicians to order whole body CT scans in trauma and its effectiveness in this regard. Scandinavian Journal of Trauma, Resuscitation and Emergency Medicine 2013 21(Suppl 1):S16. 